Aus der k. k. dermatologischen Univensitätsklinik von Prof.

F. J. Pick in Prag.

\title{
Ueber die aus weichen Naevis entstandenen bösartigen Geschwülste.
}

\author{
Von \\ Priv.-Docent Dr. Ludwig Waelsch, \\ I. Assistent der Klinik.
}

(Hierzu Taf. IX u. X.)

Die mikroskopische Untersuchung dreier aus Naevis entstandener Geschwülste gab mir Gelegenheit, der Frage über die Zngehörigkeit derselben zum Carcinom räher zu treten. In Folgendem möchte ich über die Ergebnisse berichten.

Der erste Fall betraf L. A., 56 Jahre alt, Schmiedsgattin, aufgenommen am 24./XII. 1897.

Pat. gibt an, dass sich vor einem halben Jahre aus einem Muttermale über dem rechten Schulterblatt die jetzt bestehende Geschwulst zu entwickeln begann. Seit dieser Zeit babe auch ilar Allgemeinbefinden gelitten, sie sei stark abgemagert, habe häufig Schwindel, Kopfsehmerzen, wenig Appetit. Ein consultirter Arzt verordnete ihr ein schwarzes Pfaster, nack dessen fortgesetzter Application die kleinen Fifforescenzen in der Nachbarschaft des Tumors in grösserer Zahl aufgeschossen seien. Früher war Pat. stets gesund; über das Vorkommen von Geschwülsten in ihrer Familie liess sich Nichts eruiren,

Status praesens: Die Kranke ist stark herabgekommen, kachektisch, die allgemeinen Hautdecken fahl, leicht atrophisch. An der Haut über der rechten Scapula findet sich ein annähernd spindelförmiger Tumor von einem Längsdurchmesser von ca. $10 \mathrm{Cm}$., einem queren von ca. $6 \mathrm{Cm}$, von mässig derber Consistenz und vielfach gelapptem Bau, so dass eine blumenkohlartige, tiefschwarzblaue Wucherung entsteht, die stellenweise exulcerirt und schmierig belegt, einen aashaften Geruch verbreitet. Um diesen Tumor gruppiren sich dicht beieinander stehende braunrothe oder im Beginne einer schwarzen Pigmentirung befindliche stecknadelkopf- bis 
erbsengrosse Knoten, die das Niveau der Haut mehr weniger überragen. Dieselben stehen an den dem Tumor zunächst gelegenen Hautpartien sehr dicht, während sie an der Peripherie, wo sie auch bedeutend kleiner sind, sich isolirter anordnen. Viele dieser Knoten zeigen eine ganz eigenthümliche Transparenz. Die Haut zwischen den kleinen Tumoren ist stark geröthet, mit kleinsten Pusteln besetzt.

Die unterste nucbale Drüse rechts haselnussgross, ebenso die rechtsseitigen supraclavicularen; die axillaren Drüsen rechts wenig vergrössert, links za einem wallnussgrossen Tumor verbacken.

Die Untersuchung der inneren Organe ergab normale Verhältnisse, ebenso die des Harnes. Die Blutzählung ergab Verminderung der rothen Blutkörperchen. - Während des kurzen Spitalaufenthaltes der Patientin wuchs der Tumor zusehends und zeigte fortschreitenden Zerfall, unter rapider Zunahme der Zahl der miliaren Metastasen, und unter zunehmender Kachexie der Kranken. Sie verliess über eigenen Wunsch am 12. Januar 1898 unsere Klinik in elendem Zustande. Weitere Nachrichten sind uns über sie nicht zugekommen.

Der Patientin wurde ron der afficirten Rückenhaut ein Stückchen excidirt, das zahlreiche kleinste pigmentirte und pigmentlose Tumoren aufwies. Die histologische Untersuchung der in Alkohol und Sublimat fixirten Stickchen ergab nach Einbettung in Celloidin und Paraffin das später zu beschreibende Bild. Die Schnitte wurden mit den gebräuchlichen Kernfärbemitteln tingirt, ferner wurden verwendet zur Darstellung der elastischen Fasern Ta enzer's Orceinfärbung und Weigert's neue Färbung, zux Darstellung des Bindegewebes die ausgezeichnete Färbemethode Hansen's (4).

Die Ergebnisse der histologischen Untersuchung seien in Folgendem niedergelegt: Das Deckepithel ist normal, in der basalen Zellschichte hie und da etwas stärker pigmentirt. Die Retezapfen erscheinen normal, nur dort; wo die Tumormasse direct an das Epithel sich herandrängt, sind die Zapfen und die zwischen denselben gelegenen Papillen stark abgeflacht oder ganz verstrichen, dabei ist dann das Epithel manchmal etwas verschmächtigt auf wenige Iagen abgeplatteter Zellen reducirt.

Der im Corium sitzende Tumor setzt sich zusammen aus grösseren und kleineren scharf begrenzten rundlichen Zellballen oder länglichen Zellsträngen. Die ersteren stellen zum Theile Querschnitte der letzteren dar. Die Anordnung dieser umschriebenen Zellmassen, sowie deren Elemente zeigen vollständige Uebereinstimmung mit denen eines Naerus, sie stellen in ihrer Lage- 
rung zu einander and zum Deckepithel eine vollendete Nach. ahmung des histologischen Bildes eines Naevus dar.

Diese Zellhaufen setzen sich zusammen aus Elementen, welche deutlich den Charakter ron Epithelabkömmlingen tragen. Zelle an Zelle derselben Art ist unmittelbar aneinandergereiht, es sind grosse protoplasmareiche Zellen mit grossem, rundlichem bis längsovalem, granulirtem Kern, die hie und da ebenso pigmentirt sind wie die Epithelzellen der Epidermis (cfr. Scheuber (3) p. 184), In den centralen Theilen mancher, besonders der grösseren Ballen finden sich geringe Mengen von Rund- und Spindelzellen, die jedoch nie so reichlich auftreten; dass sie das Gepräge des Tumors als eines aus Naevuszellen entstandenen rerwischen könnten. Sie verdanken ihre Entstehung einer gleichzeitigen Wucherung des Bindegewebes. In den Ballen selbst, besonders an deren Peripherie, lassen sich sehr zahlreiche Mitosen nachweisen.

Neben den grossen Zellhaufen finden sich zallreiche kleinere, durch eine schmale Bindegewebsbrücke vou denselben getrennt, oder woch mit dem Mutterballen in Verbindung.

Die Ballen und Stränge begrenzen sich zumeist gegen das Bindegewebe sehr scharf, an anderen Orten findet aber ein mehr allmäliger Uebergang statt, indem der Ballen an seiner Peripherie sich weniger compact zeigt, und als eine mehr diffuse Einwucherung gegen das Bindegewebe vordringt. Dort, wo die grösseren Ballen sehr scharf demarkirt sind, ist das Bindegewebe bäufig zu einer förmlichen Hülle verdichtet, die in Form der Länge nach aneinandergelagerter spindeliger Zellen den Tumor gegen das lockere Bindegewebe der Nachbarschaft abschliesst. Von dem benachbarten Bindegewebe lassen sich dann feine Züge in die Ballen hinein verfolgen, die dort, wo sie sich enger durchflechten, dem Tumor einen deutlich alveolären Bau verleihen, wie die Hansen'sche Färbung sehr schön zeigt. Dieselbe erweist auch die Intactheit des subepithelialen Bindegewebes, das nur dann verschwindet, wenn der Tumor direct an das Deckepithel herangerückt ist, wobei dann erentuell die obersten Schichten des Tumors sich direct anschmiegen an das verschmächtigte auf wenige Zellreihen roducirte Deckepithel.

An den Ballen, hauptsächlich an deren dem Deckepithel 
zugewendeten Peripherie, finden sich zahlreiche ron goldgelben kleinsten Pigmentkörnchen erfüllte Zellen. Die Hauptmasse des Tumors sitzt. in den oberen Coriumschichten in Form der oben beschriebenen Ballen und Stränge, in den tieferen Schichten sind gleicbartige, grössere und kleinere Zellanbäufungen verstreut.

Die Geschwulstmasse substituirt alle Gewebe des Ortes ihrer Entwickling; das Bindegewebe und die elastische Fasern, die Anluangsgebilde der Haut. Haarbälge und deren Talgdrüsen, theilweise auch die Schweissdrüsen, die Blutgefässe sind vollständig zu Grunde gegangen. Letztere finden sich nur in den gröberen bindegewebigen Septis zwischen den Ballen, ron denen aus feine Capillaren mit den die grösseren Ballen selbst septirenden Bindegewebszïgen in die Tumormasse sich hinein verfolgen lassen. Mit Hilfe der elastischen Faserfärbung (Taenzer, Weigert) lassen sich in den grösseren und spärlicheren Ballen der tieferen Coriumschichten Reste von ein Lumen umgrenzenden elastischen Fasern nachweisen, die nach Art der elastischen Fasern der widerstandsfähigen Intima aneinander gelagert sind. Dieser Befund, zusammengehalten mit der Localisation der Zellballen 'an Stellen, wo die Blutgefässe zu suchen wären, lässt den Gedanken nicht von der Hand weisen, dass diese Metastasen ibren Ausgangspunkt genommen ron Blutgefässen.

Im zweiten Falle handelte es sich um einen $40 j a ̈ h r i g e n$ Taglöhner. Aufgenommen am 9. November 1887.

Patient gibt an, er hätte an der Stelle, wo jetzt die Geschwulst an der Bauchbaut sitze, seit Jahren einen glatten, schwarzbrannen Fleck besessen, dessen Grösse die eines jetzt noch vorhandenen am äusseren Theile der linken vorderen Achselfalte übertroffen hätte. (Die Grösse des letzteren Fleckes beträgt 1-2 $\mathrm{Cm}$. im längsten und $3 / 4 \mathrm{Cm}$. im kürzesten Durchmesser.) Auf dem Fleck der Bauchhaut sei nun, ohne dass er selbst sich in der Fläche vergrössert hätte, eine schwarze, warzenartige Wucherung entstanden, aus welcher im Verlaufe eines halben Jahres der jetzige Tumor sich entwickelte. Gleich zu Beginn des Wachsthums der Gesebwulst wurde dieselbe in Folge der Reibung der Kleidungsstäcke an der Oberfläche erodirt, wobei wiederbolte mässige Blutungen stattfanden. Die Drüsengeschwulst links in inguine besteht angeblich seit 6 Monaten, und entwickelte sich allmälig nach dern Beginne des Wachsthums der Geschwulst an der Bauchhaut. Näheres über die Art und-Raschheit des Wachsthums 
Ueber die aus weichen Naevıs entst. bösartigen Geschwülste. 253

der Drüsengeschwulst vermag der Lranke nicht anzugeben. Er führt ihre Entstehung zurück auf eine Abschürfung am linken Fusse, die er sich bei einem langen Fussmarsch zugezogen.

Status praesens: Patient ist kräftig gebaut, von gutem Ernährungszustand. An der Haut des Stammes und der Extremitäten finden sich zahlreiche, verschieden grosse, dunkelpigmentirte flache Naevi.

Die Geschwulst an der vorderen Bauchwand zeigt in ihrem linken grösseren Antheile dunkelschwarze Oberfläche, ist höckerig, aus kleineren Lappen zusammengesetzt und grenzt sich sowohl nach der Tiefe, wie nach den Seiten gegen die gesunde, nicht verfärbte Haut scharf ab. Mit. einem schmalen Fortsatz, über welchem die Haut eine leichte dunkle Verfärbung zeigt, zieht sie gegen die Medianlinie.

Die Geschwulst in inguine setzt sich zusammen aus mehreren kleinen Tumoren (Drüsen), von verschieblicher Haut bedeckt, und in toto über der Unterlage verschieblich. An der deckenden Haut ein linsengrosser, dunkelbrauner Pigmentfleck, ähnlich den sonstigen, unregelmässigüber den Körper vertheilten.

Von der Geschwulst an del Bauchwand, die operativ entfernt wurde, sowie ron den exstirpirten Driisen standen mir nun einige Stücke zur Verfügung, deren mikroskopische Untersuchung folgendes Bild ergab:

1. Kleines, mässig pigmentirtes Hautstückchen mitglatter Oberfläche.

Das Deckepithel ist dort, wo die Tumorbildung auf ihrer Höhe erscheint, stark verdünut, und fehlt an den exulcerirten Stellen vollständig. In der basalen Zellschichte lässt sich überall ungemein reichliches Pigment nachweisen, das entweder als rundlicher braunschwarzer Pigmentballen die ganze Zelle ausfüllt. so dass der Zellkern verdeckt wird, oder als feinkörniges Pigment in dem Zellleib verstreut ist. Auch zwischen den Zellen des Stratum mucosum, ja sogar in den Hornschichten lassen sich allenthalben, besonders dort, wo die basalen Zellschichten sich schon in Tumorgewebe nmzuwandeln beginnen, rundliche oder verästelte pigmeutirte Zellen nachweisen.

An jenen Stellen, wo das Deckepithel verdünnt ist, sind die Retezapfen verschwunden, an anderen Stellen wiederum, nämlich dort, wo sie förmliche Septa zwischen den Ballen des Tumorgewebes bilden, sind sie oft mächtig gewuchert, gabelig getheilt und wandeln sich dabei ihre Zellen direct urn in die der Neubildung. An diesen rerlängerten Zapfen füllt zuerst auf ihr ungemein grosser Pigmentreichthum. Das Pigment ist 
auch hier hauptsächlich gebunden an die basalen Zellen, aber auch unregelmässig vertheilt über die ganze schmale Fläche des Epithelzapfens in Form dunkelbrauner pigmentirter Zellen oder feinkörnigen intracellulären Pigmentes. Die Zellen dieser Zapfen sind in der Längsrichtung des Zapfens abgeplattet, ihre Kerne längsoval oder spindelig; bei stark verschmälerten Zapfen, die nur aus 2-3 Zellagen bestehen, sind die dem Bindegewebe oder dem in dasselbe eingelagerten Tumor zunächst gelegenen nach Art von Aehrengrannen aufgelockert, wobei die freien Enden der Zellen nach abwärts sehen.

Zwischen dem Deckepithel und dem Tumorgewebe, das in das Corium in Form scharfbegrenzter Balken und Ballen, bestehend aus deutlich epithelialen Zellen, eingelagert ist, lassen sich Uebergänge constatiren. An diesen Stellen, wo der Epithelzapfen direct sich fortsetzt in das Gewebe der Geschwulst, verdeckt oft die kolossale Ansammlung des tief braunen Pigmentes das histologische Bild vollständig, und bezeichnet nur ein grosser Pigmentballen den Ort dieser Metaplasie. An anderen Stellen aber lässt sich dieser Uebergang deutlich beobachten. Die längsovalen oder spindeligen Zellen des Zapfens nehmen wieder mehr rundlichere Contour an, zeigen einen sich schön färbenden fein granulirten Kern mit deutlichem Protoplasmaleib, mit der Tendenz dieser Zellen sich zu kleineren und grösseren Ballen und Strängen zu vereinigen, die in ihrem Bau vollständig übereinstimmen mit dem eines Naevus. Die einzelnen Zellen dieser Ballen sind aber auch nicht ganz gleichmässig gebaut, indem man neben solchen mit rundem bläschenförmigem Kern auch solche mit ovalem oder direct spindeligem Kern' erkennt.

Diese Zellhaufen finden sich aber nicht nur im Corium und zwar im Gegensatz zum vorigen Tumor tief hineinwuchernd in die, Cutis, sondern auch in Form kleiner scharf begrenzter Ballen im Deckepithel, und zwar sowohl eingelagert zwischen die basalen Zellschichten als auch zwischen die höheren des stratum mucosum.

Diese kleineren, oft auffallend stark pigmentirten Nester sind allseits rom Epithel umgeben, das sich gegen diese Zellanhäufungen allmälig abplattet, so dass sie scharf umgrenzt er- 
scheinen von einem schmalen Saum spindeliger Zellen. Sitzen sie in den untersten basalen Zellschichten, so Iässt sich am unteren Rande gegen das Bindegewebe des Corium eine schmale treunende. Zone von basalen Zellen nachweisen.

Bezüglich der Vertheilung des Bindegewebes zwischen den einzelnen Ballen und Strängen des Tumors, sowie in demselben ferner der Anordnung der elastischen Fasern, der Blutgefässe zeigen sich dieseiben Verhältnisse wie in den kleineren metastatischen Tumoren des ersten Falles. Es zeigt auch dieser Tumor das typische Bild des Naerus, jedoch mit excessiver Zellwucherung.

Die Vertheilung des Pigmentes in den einzelnen Zellnestern, speciell denen der tieferen Coriumschichten, ist eine sehr ungleichmässige. Manche Zellstränge sind ganz frei von Pigment, andere lassen dasselbe nur an ihrer Peripherie erkennen und zwar in Form länglicher oder schmaler spindeliger Pigmentzellen in dem die Ballen trennenden Bindegewebe, hier oft auffallend reichlich um die im Bindegewebe ziehenden Gefässe. Sind sie in grosser Zahl in den bindegewebigen Septis gehäuft, so legen sie sich der Länge nach aneinander und bilden durch ihre dunkelbraune Farbe scharf herrortretende Septa, welche, seitliche Zweige aussendend, den grossen Zellhaufen in kleinere Nester auflösen. Ausserdem findet sich auch Pigment zwischen den Zellen des Tumors selbst und in demseiben; im letzteren in Form feiner dunkelbrauner Körnchen. In den tiefsten Theilen des Tumors ist die Pigmentanhäufung direct eine ungeheure, das Gewebe der Geschwulst ist hier vollständig verdeckt durch dunkelbraune, fast schwarze Pigmentzellen und unregelmässig begrenzte grosse Pigmentschollen.

2. Kleines Hautstückchen nitziemlich glatter Oberfläche,das schoumakroskopischinder Tumormasse grösseredunkelbraunschwarze, durchlichtere Septa ron einander getrennte Knoten erkennen lässt.

In Uebereinstimmung mit diesem makroskopischen Befund war auch das herrorstechendste Moment im mikroskopischen Bilde eine ungemein starke Pigmentirung, welche fast rollständig das feinere histologische Bild verdeckte, ferner die Bildung 
scharf begrenzter grosser Tumorknoten, die ron verschiedener Grösse entweder bis knapp an das Deckepithel reichten, oder einen mehr weniger breiten Saum von Bindegewebe zwischen sich und dem Epithel frei liessen, und andererseits weit hineinreichten in das Unterhautzellgewebe.

Auch hier liessen sich directe Uebergänge rom Deckepithel in die Tumormasse nachweisen, unter allmäliger Veränderung des Charakters dieses Epithels, und der Lagerung seiner Elemente gegeneinander. Auch hier finden sich ansserdem, wie bei dem ersten Hautstïckchen dieses Falles, im Deckepithel umschriebene, scharfbegrenzte Zellnester, deren Elemente nicht pallissadenförmig aneinander gereiht sind, wie die ihrer Nachbarschaft, sondern unregelmässig aneinander liegen, stellenweise eiue Andeutung concentrischer Schichtung, erkennen lassen. Diese kleinen Ballen sind oft so stark pigmentirt, dass nur ein schwarzer, intraepithelial gelegener Fleck ihren Sitz anzeigt. Das Pigment ist auch hier nicht ausschliesslich an das Tumorgewebe gebunden, sondern findet sich wie in dem ersten Hautstückchen in Bindegewebe, speciell in dem der Papillen in Form rundlicher, zugespitzter, oder verästelter Pigmentzellen in einer dem Blutgefässverlauf in den Papillen entsprechenden Richtung und Anordnung, ferner auch unregelmässig verstreut im subpapillären Bindegewebe.

Auch in den sonstigen Gewebsveränderungen zeigt der Tumor vollständige Uebereinstimmung mit den durch die Untersuchung des ersten Hautstïckchens gewonnenen Resultaten.

Von diesem Falle standen mir auch exstirpirte Lymphdrüsen zur Verfügung. Die Untersuchung derselben ergab keine pathologischen Veränderungen.

Fall III. Der dritte von mir untersuchte Tumor stammte von einem 30jährigen Manne und hatte sich auf dem Boden eines Nävus der Brusthaut imnerhalb dreier Monate allmälig entwickelt.

Er stellte dar eine pilzförmig sich erhebende Wucherung, die durch einen schmäleren Stil mit der Brusthaut in Verbindung stand. Seine Oberfläche in der Mitte ziemlich glatt, stellenweise exculcerirt; an den Randpartien haben sich kleine Tochtertumoren gebildet, die aus kleineren Knoten bestehen, wodurch Maulbeer-oder Himbeerformen zu Stande kormen. 
Der Tumor selbst ist nicht auffallend pigmentirt. Beim Durchschneiden in senkrechter Richtung zeigt sich aber seine dem Stil entsprechende Basis zur Hälfte tief schwarz gefärbt.

Der mikroskopischen Untersuchung wurde unterzogen: ein Stück der maulbeerförmigen Tumoren, eines der mit glatter Oberfläche ausgezeichneten pilzförmigen Wucherung, endlich ein Theil des schwarz pigmentirten Stiles.

1. Maulbeerförmiger Tumor: Derselbe ist allseits umschlossen von einer mehrschichtigen Lage von schönem Deckepithel, das sich von normalem nur durch seine spärlichen niedrigen Zapfen unterscheidet. Vom Grunde der tiefen Epitheleinsenkungen, welche die einzelnen Lappen des Tumors von. einander trennen, gehen ungemein dünne und zarte, oft verzweigte Epithelzapfen weit in das Tumorgewebe hinein.

Der Tumor selbst zeigt bezüglich der ihn zusammensetzenden Zellelemente denselben Bau wie die vorherbeschriebenen Geschwülste, nämlich Zellen von deutlich epithelialem Charakter, und auch das Pigment ist in derselben Weise im Tumorgewebe vertheilt.

Dagegen ist die Anordnung der Zellen eine andersartige. Es finden sich nämlich scharfumschriebene, nach Art der Naevuszellen aneinandergelagerte Zellnester nur sehr spärlich und da zumeist in der Nähe des Deckepithels, manchmal umgeben von einer förmlichen Hülle dicht aneinandergelagerter, spindelförmiger, sehr stark pigmentirter Bindegewebszellen.

Die Hauptmasse der Geschwulst wird vielmehr gebildet von dicht aneinanderliegenden Zellen, die in mit Kernfärbemitteln tingirten Schnitten ganz gleichmässig angeordnet sind. Bei Bindegewebsfärbungen (Hansen) hingegenzeigt sich, dass der Tumor, so wie die früher beschriebenen, einen deutlich alveolären Bau besitzt, indem die scheinbar gleichmässige Tumormasse sich auflöst in kleine und kleinste Ballen ron Naevuszellen, die durch zarteste bindegewebige Septa von einander getrennt sind.

Besonders auffallend ist auch der Reichtbum dieses Tumors an Blutgefässen, die sowohl, von zartem Endothel umgeben, sich in der Geschwulstmasse selbst verzweigen, als auch 
in Form grösserer Capillaren im. Bindegewebe zwischen den einzelnen Ballen.

2. Stück des Tumors mit glatter, stellenweise exulcerirter $O$ berfläche.

Dieser Geschwulstform eigenthümlich ist eine in grösseren Abständen ungemein stark entwickelte Tiefenwucherung des Deckepithels, das spärliche, sehr lange, aus wenigen Lagen spindeliger, stärkst abgeplatteter Zellen bestehende dünne Zapfen in die Tiefe entsendet, welche den ganzen Tumor in mehrere kleinere theilen. Im Bereiche der exulcerirten Partie entbehrt er des Deckepithels und zeigt hier auch noch über die exulcerirte Stelle hinaus einen so grossen Reichthum an Rundzellen, dass der epitheliale Charakter der Geschwulst ganz verwischt wird. Die jenseits dieser entzïndlich infiltrirten Geschwulstpartien gelegene Tumormasse zeigt das eben beschriebene Bild der nur hie und da von Naevusballen unter. brochenen gleichmässigen Zellanhäufung, die erst bei Bindegewebsfärbungen ihren alveolären Bau erkennen lässt. Im Bereiche der von Rundzellen stark durchsetzten Partie fehlen aber zumeist die feinen Bindegewebsfasern, so dass hier die Tumormasse auch nach diesen Färbungen einen ganz gleichmässigen. Clarakter zeigt.

In den tieferen Partien des Tumors treten neben den Geschwulstzellen von gewöhnlichem Bau solche mit sehr grossem, schön gefärbtem Kern hervor, welche oft im Innern Vacuolen zeigen.

Sehr gross ist der Reichthum an Blutgefässen, besonders in den von der Entzündung weniger heimgesuchten Partien zunächst dem Deckepithel, und dann wieder in dessen tieferen Partien, während die mittleren, stark von Rundzellen durchsetzten Theile zwar auch noch reichlich vascularisirt sind, aber doch nicht so hochgradig wie die vorerwähnten Theile. An diesen ist der Reichthum an Blutgefässen ein solcher, dass dieselben ein förmliches Stroma bilden für die zwischen sie eingelagerten Geschwulstzellen. An diesen Stellen ist auch der Gehalt an goldgelbem Pigment ein ungemein auffallender.

Das dritte Stückchen wurde der Tumorbasis entnommen, jenem Stil, durch welchen die pilzförmige Wucherung 
mit dem in der Haut gelegenen Geschwulstgrund zusammenhing. Derselbe zeigte sich zur Hälfte tiefschwarz pigmentirt und schnitt diese Pigmentirung in einer scharfen senkrecht verlaufenden Linie gegen die weisse Tumormasse $a b$ und begrenzte sich nach oben in bogenförmigem Contour gegen die pilzförmige Wucherung.

Der pigmentirte und der nicht pigmentirte Theil ergaben nun ein verschiedenes histologisches Bild.

Der weisse nicht pigmentirte Theil zeigte einen den früher beschriebenen analogen $\mathrm{Bau}$, eine gleichmässige Zellanhäufung, Zelle an Zelle derselben Art, hie und da eine von exorbitanter Grösse und verschiedenartig gestaltetem Kern. Dazwischen Züge von Spindelzellen, in deren Bereich auch eine starke Anhäufung von Rundzellen sich constatiren liess. Der Tumor selbst in grössere Ballen geformt, welche in seinen tieferen Sclichten durch ihre besonders scharfe bindegewebige Begrenzung und den Rest von Fettgewebe an der Peripherie sich erkennen liesseu als rom Geschwulstgewebe substituirte Läppchen des Unterhautfettgewebes. Die Färbung nach Hansen zeigte ein dichtes Geflecht von Bindegewebsfasern in der die Lappen des Tumors umscheidenden Hülle, von welcher aus spiralig gerollte spärliche Bindegewebsfasern in die Tumormasse selbst hineingingen. Im Tumor selbst sind hier nur die gröberen Faserbündel erhalten, die feinen Fäserchen lassen sich nicht nachweisen.

Die Untersuchung des pigmentirten Theiles ergab hingegen ein ganz abweichendes Bild, dessen Grundform jedoch auch hier in dem alveolären Bau der Geschwulst gegeben war, wobei die eigentlichen Geschwulstelemente auch hier epithelialen Charakter trugen. - B.- Besonders auffallend waren einerseits die Septa, andererseits die in dem ron den Septis gebildeten Maschenwerk enthaltenen Zellen. Diese Septa sind ungemein breit, und setzen sich zusammen aus vielfach miteinander verflochtenen feinsten Bindegewebsfasern, die ihrerseits ein feines, von kleinsten Capillaren durchzogenes Netzwerk bilden, dessen Maschenräume von Rundzellen durchsetzt sind. Zwischen den Rundzellen finden sich allenthalben deutliche Spindelzellen. Die von den breiten Septis umgrenzten Maschen enthalten locker 
angehäuft grosse Zellen, deren Leib fast vollständig von dem grossen Kerne eingenommen wird. Diese Kerne sind rundlich, spindelig, dreieckig, oder ganz unregelmässig geformt, enthalten oft Vacuolen, und nehmen den Farbstoff verschieden stark auf, indem sich neben blassen sehr satt tingirte vorfinden. Die Zellen liegen entweder ganz locker aneinandergelagert in den Maschen, oder bedecken deren Innenwand in einschichtiger Lage, förmlich nach Art eines Epithels, wobei sich dann noch im Lumen spärliche Kerne nachweisen lassen.

Der Tumor selbst ist nun auch von grösseren und breiten Streifen Bindegewebes durchzogen, das grössere Blutgefässe führt. In der Nachbarschaft dieser Bindegewebszïge werden die Alveolen des Tumors immer kleiner und können eudlich vollständig verschwinden, so dass dann an dieser Stelle sich bloss ein Rundzelleninfiltrat vorfindet. In diesem zeigt auch die Hansen'sche Färbung kein Binlegewebsnetzwerk mehr, sondern es finden sich nur mehr spärliche, ganz unregelmässig verlaufende Fäserchen. Im Tumor selbst ist ungemein reichliches Pigment.

Das der Geschwulstmasse benachbarte Gewebe weist die Symptome heftiger Entzündung aut in Form reichlicher dichter perivasculärer Infiltration, in welcher tietbraunschwarze Pigmentzellen in grosser Menge eingelagert siud. Dieselben finden sich auch in den grösseren bindegewebigen Septis sehr reichlich um die Blutgefässe herum. Auch um die Schweissdrüsen besteht starke Infiltration, die sich auch in Form von aus Rundzellen zusammengesetzter Septa in die Läppchen des Fettgewebes hineinerstreckt als Ausdruck einer perivasculären Infiltration um die von den grösseren Gefässen der bindegewebigen Hülle der Läppchen ausgehenden Capillaren.

Wir hatten es also in den untersuchten drei Fällen von aus Naevis entstandenen malignen Geschwülsten zu thun mit Tumoren, deren Zellen vollständige Uebereinstimamung zeigten mit den Zellen der benignen Muttergeschwulst. Da diese letztere Epithelabkömmlingen ihre Entstehung verdankt, so müssen wir auch die Zellen des aus ihr entstandenen Tochtertumores als Epithelderivate ansprechen und können sie demgemäss nicht als Sarcome, als Bindegewebsgeschwülste, bezeichnen, wir 
Ueber die aus weichen Naevis entst. bösartigen Geschwülste. 261

müssen sie vielmebr unter die epithelialen Geschwülste einreihen.

Die Stellung dieser alveolären Tumoren mit ihrem bindegewebigen Stroma und dem zelligen Inhalt der Stromamaschen im pathologisch-anatomischen System war ja seit Virchow's die Sarcome präcisirender Definition eine etwas schwankende. Beweis dessen die Benennung dieser alveolären Tumoren, als Sarcoma carcinomatodes, durch Rindfleich (8) der sie als carcinomatöse Degeneration des Sarcomes betrachtet, Beweis dessen das freimüthige Geständnis Billroth's: (9) "Diese im Ganzen seltene Geschwulstform (das Sarcoma alveolare) ist sehr schwer anatomisch zu charakterisiren. Sie kann wegen Grösse und Anordnung der Zellen dem Carcinom stellenweise so ähnlich sein, dass ich mich nicht getrauen möchte, jedes mir unter dem Mikroskope vorgelegte Stück aus einer solchen Geschwulst sofort richtig zu deuten." -

Damit soll aber nicht die Möglichkeit der Existenz walirer alveolärer Sarcome bestritten werden, sondern nur dem Bestreben Ausdruck gegeben werden, eine Gruppe dieser Tumoren, nämlich die aus weichen Naevis entstandenen, auszuscheiden und sie den Epithelgeschwülsten einzureihen.

Ich stimme hierin mit Un na (1) und Hod ar a (2) rollständig überein, und glaube auch durch die Schilderung des histologischen Bildes die Einwände von Ledham Green (7) und von Bauer (8) genügend entkräftet zu haben. Speciell der directe Uebergang des Deckepithels in das Tumorgewebe, die Einlagerung von Geschwulstelementen in das erstere, wribei Täuschungen des mikroskopischen Bildes, wie sie Bau er zur Stützung seiner Anschauung heranzieht, vollständig ausgeschlossen sind, beweisen den epithelialen Ursprung dieser Tumoren. Auch der Einwand von Green, dass die Chorioidealsarcome, deren Entwicklung auf bindegewebigem Wege bisher unbestritten ist, in Architektur und Structur vollständige Uebereinstimmung zeigen mit den sogenannten aus Naevis entstandenen Sarcomen, erscheint mir nicht ganz berechtigt, bevor nicht eine neuerliche Prüfung dieser Tumoren nach der strittigen lichtung erfolgt ist.

Es ist ja sicher, und die umfassende Monographı von 
Fuchs (9) beweist dies, dass Sarcome, und zwar Spindelzellensarcome in der Chorioidea aus der Schichte der grossen Gefässe ihren Ursprung nehmen, ebenso sicher ist aber, dass, wie auch aus der erwähnten Arbeit von $\mathrm{F} u \mathrm{ch}$ s an mehreren Stellen ${ }^{1}$ ) ersicbtlich ist, dass ein grosser Theil der alveolären Tumoren in seinen Zellen epithelialen oder wenigstens Epithel sehr ähnlichen Charakter zeigt. So hebt Fuchs (p. 75) hervor: „Die in den Lücken des alveolären Maschenwerkes liegenden Sarcomzellen sind gross, rundlich oder polygonal, besitzen einen grossen meist ovalen Kern mit einem sehr grossen und deutlichen Kernkörperchen. Diese Zellen, welche einen dem Epitbel sehr ähnlichen Habitus haben, bilden bald kleinere Gruppen, bald grössere Conglomerate. . . . . Von den Balken des Gerüstes sieht man hin und wieder feinste Fäserchen zwischen die im Maschenraum liegenden Zellen hineingehen, so dass hier eine Art feinsten, aber sehr unvollständigen Reticulums entsteht. ...W enn man aber von diesem rudimentären Reticulum, das überhaupt nur schwer und nicht iberall nachzuweisen ist, absieht, bieten diese Theile des Tumors genau das Bild eines Carcinomes dar."

Es ist ja denkbar, dass auch diese der Chorioidea entstammenden alveolären Tumoren ihren Ursprung nehmen aus in die Chorioidea versprengten Keimen des retinalen Pigmentepithels, und anch die Beobachtung der Entstehung bösartiger melanotischer Tumoren aus pigmentirtem Naevis der Iris $^{2}$ )

l) 1. c. p. $67,72,75$.

2) Fuchs 1. c. p. 234. "Es sind zweí Falle bekannt (Hirsebberg, Warren Tay), wo sich Sarcome der Iris aus lange bestehenden, wahrscheinlich angeborenen Pigmentflecken entwickelten, in ähnlicher Weise, wie z. B. melanotische Warzen der Haut sarcomatös werden können. Ferner berichtet $\mathrm{H} u l k$ e über eine Frau, bei welcher seit der Geburt eine sebr dunkle Pigmentirung der Haut an der Augenbrane und den Lidern, sowie eine Anzahl schwarzer Flecke auf der Sklera des rechten Auges bestanden hatten. Als die Frau 62 Jahre alt war, fing das Sehvermögen des rechten Auges zu sinken an. Man fand eine schwarze Masse vom Augenhintergrunde vorspringen. Das Auge wurde später enucleirt und enthielt ein Melansarcom im hinteren Abschnitt der Adnexhaut. Die schwarzen Flecke in der Sklera erwiesen sich als einfache Pigmentationen." 
Ueber die aus weichen Naevis entst. bösartigen Geschwülste. 263

lassen mir die Genese aller Chorioidealsarcomen als nicht so ganz sicherer scheinen, wie es Ledham Green annimmt. ${ }^{1}$ )

Wir hatten es also in den untersuchten Fällen zu thun mit epithelialen Geschwülsten, die sich entwickelten aus präexistirenden Anlagen epithelialer Natur, die dann in dem Aussehen und dem Aufbau der zelligen Elemente vollständige Uebereinstimmung zeigten mit dem schon ron Haus aus pathologischen Mutterboden, aus dem sie sich entwickelten.

Es stellen demnach diese aus embryonalen oder in frühester Jugend abgelagerten Keimen entstandenen Tumoren einen schönen Beweis dar für die Richtigkeit der $\mathrm{C}_{0} \mathrm{~h} n \mathrm{~h}$ e i m'schen Theorie über die Aetiologie mancher Geschwülste. Und wenn Cohnheim (10) bei Erörterung seiner Theorie schreibt: „Das Einfachste erscheint mir zweifellos, sich vorzustellen, dass in einem frühen Stadium der embryonalen Entwicklung mehr Zellen producirt werden, als für den Aufbau des betreffenden Theiles nothwendig sind, so dass nun ein Zellquantum übrig geblieben ist von an sich nur sehr geringfügigen Dimensionen, aber - wegen der embryonalen Natur seiner Zellen — von grösserer Vermehrungsfähigkeit"; und weiter: "möglich ferner, dass das überschüssige Zellmaterial ... an einer Stelle abgeschlossen sitzen bleibt: dies würde die locale Anlage zur späteren Geschwulstbildung involviren", so scheinen diese Worte förmlich auf die Naevusgeschwülste gemünzt zu sein, und es ist nur zu bedauern, oder wie Unna es treffend nennt, eine "Ironie des Schicksals", dass Cohnheim nicht die epitheliale Natur des Naevus erkannt hat, denn eine bessere Stïtze seiner Theorie hätte er nicht finden können.

Zum Schlusse fühle ich mich verpflichtet, meinem verehrten Chef, Herrn Professor F. J. Pick, meinen besten Dank für die Unterstützung dieser Arbeit auszusprechen.

I) Vgl. auch Wintersteiner (11), der an Pigmentflecken der Conjunctiva den histologischen Bau des weichen Naevus constatiren konnte, und auf die Hänfigkeit der Entstehung von "Sarcomen ${ }^{4}$ aus diesen Naevis hinweist. 


\section{Literatur.}

1. Unna. Histopathologie der Hautkrankheiten, p. 746. - 2. Hodara M. Das Verhalten der Epithelfaserung während der Entwicklung der weichen Muttermäler und des alveolären Carcinom. Monatsh. f. prakt. Derm. 1897, p. 205. - 3. S cheuber A. Ueber den Ursprung des weichen Naevi, Arch. f. Derm. u. Syph. (Festsch. f. F. J. Pick, Bd. II.) p. 175. -4. Hansen. Anatom. Anz. 1898. - 5. Rin d fleis ch. Pathol. Gewebslebre, 3. Aufl. p. 107. - 6. Bill roth. Allgem. Chirurgie, 1885, p. 835. - 7. Green Ledham. Ueber Naevi pigmentosi und deren Beziehung zum Melanosarcom. Virchowa Archiv Bd. 134, 1893, p. 331. - 3. B a uer C. Ueber endotheliale Hantwarzen und deren Beziehungen zum Sarcom. Virchows Archiv, Bd. 142, 1895, p. 407. -- 9. Fuchs E. Das Sarcom des Noealtractus, Wien, 1882, bei Braumüller. - 10. Cohnheim. Vorlesungen über allgemeine Pathologie, Berlin, 1877, bei Hirschwald, p. 635 . - 11. Winterstein $H$. Beobachtungen und Untersuchungen über den Naevus und das Sarcom der Conjunctiva. Bericht über die 27. Versammlung der ophthalmolog. Gesellschaft, Heidelberg, 1898.

\section{Eriklärung der Abbildungen auf Taf. IX u. $\mathbf{Z}$.}

Fig. 1. Schnitt durch ein Stück des Tumors von Fall II. Ocul. III. Obj. IV. Reichert Hämatoxilinfärbung. Scharf umschriebener Zellballen in den tiefen Schichten des Deckepithels mit schönen, runden Kernen. Die Zellen vollständig analog gebaut den Naevuszellen, sowie denen der Tumorballen. Zwischen und in den einzelnen Zellen feinkörniges, goldgelbes Pigment. Die Begrenzung dieser Zellballen gegen das Deckepithel ist, stellenweise vicht sehr scharf, indem sich deutliche Uebergänge der Zellen des Deekepithels in die des Tumorgewebes constatiren lassen.

Fig. 2. Dasselbe Tumorstückchen; gleiche Vergrösserung und Färbung. Nester von Tumorzellen in den basalen Epithelschichten. Directer Uebergang des Deckepithels in die (im Bilde nicht mehr sichtbaren) tieferen Tumorschichten durch einen stark pigmentirten Epithelstreifen, an den seitlich Tumorzellballen herantreten.

Fig. 3. Schnitt durch ein kleines Knötchen der Metastasen des Falles I. Färbung nach Hansen. Der Tumor zeigt das mikroskopische Bild eines typischen Naevus. Die grösseren Zellballen begrenzen sich sehr scharf durch Bindegewebsfasern die spärliche Ausiäufer in die Zellen. nester entsenden, und innerhalb derselben hie und da kleinere Zellcomplexe umgrenzen.

Fig. 4. Schnitt durch einen grösseren Knoten (Metastase) des Falles I. Methylenblau-Orceinfärbung, Der grosse Knoten Jässt seine Entstehung aus klejneren Ballen von Zellen deutlich erkennen. Zwischen den kleinen Ballen hie und da spärliche, elastische Fäserchen. In der Septis zwischen den grossen Tumorballen die elastischen Fasern spärlich erbalten, mit wenigen zarten Fäserchen zwischen die Zellbaufen der Ballenperipherie sich fortsetzend.

Fig. 5. Schnitt durch den blumenkohlartigen Theil des Tumors von Fall IIl. Färbung nach Hansen. Das Deckepitbel lange Fortsätze in die Tumormasse entsendend. Das Tumorgewebe selbst, reichlich vascularisirt, setzt sich zusammen aus dicht aneinandergelagerten kleineren Ballen, die durch zarteste Bindegewebsfasern sich scharf gegeneinander begrenzen.

Fig. 6. Derselbe Tumor. Kernfärbung (Cocbenillealaun). Das Deckepithel entsendet einen langen Zapfen in dic Tiefe, dem ein seharfbegrenzter Zellballen anliegt. Sonst das sehr reichlich vasicularisirte Tumorgewebe ganz gleichmässig gebaut. Die Anordnung in Zellhaufen bis auf den einen vorerwähnten nicht sichtbar. 

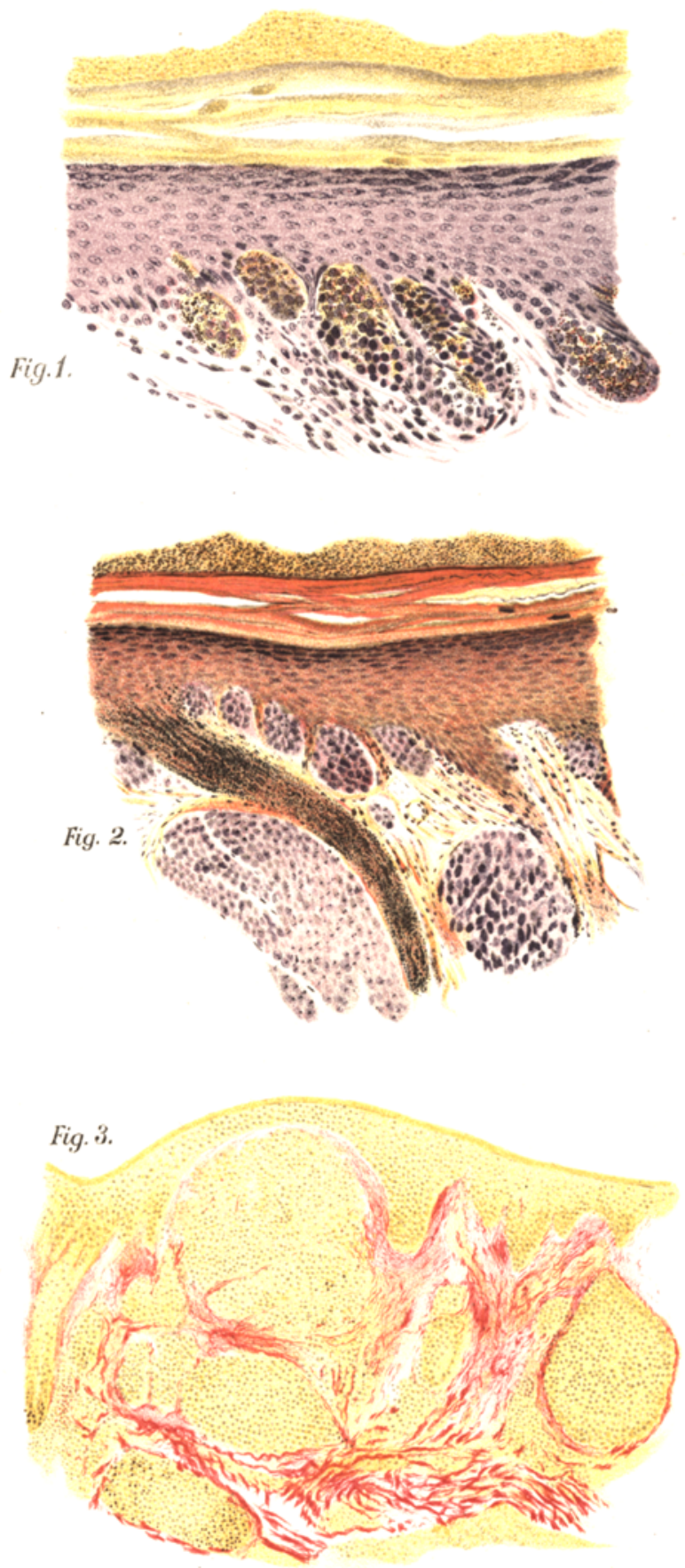


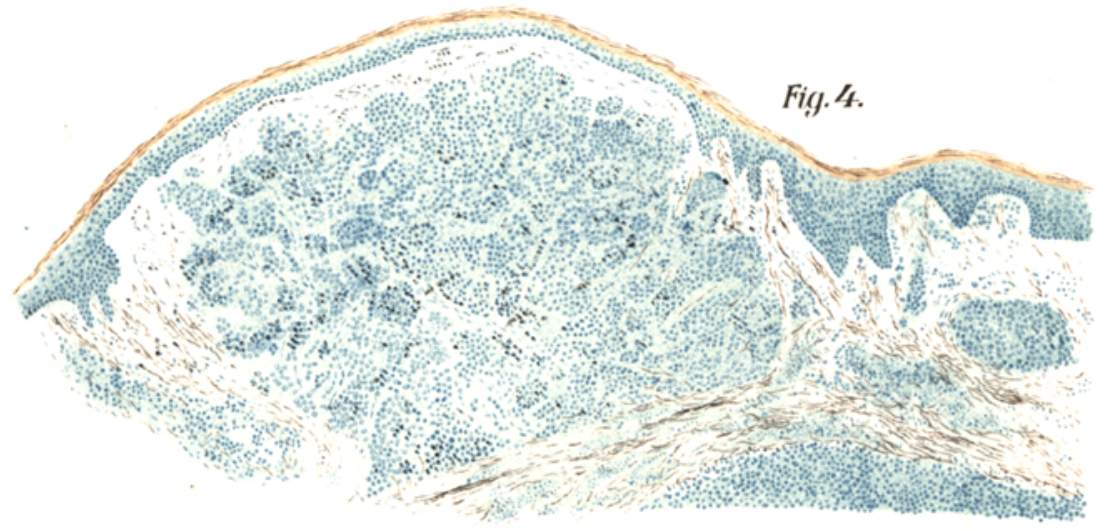

Fig. 5.
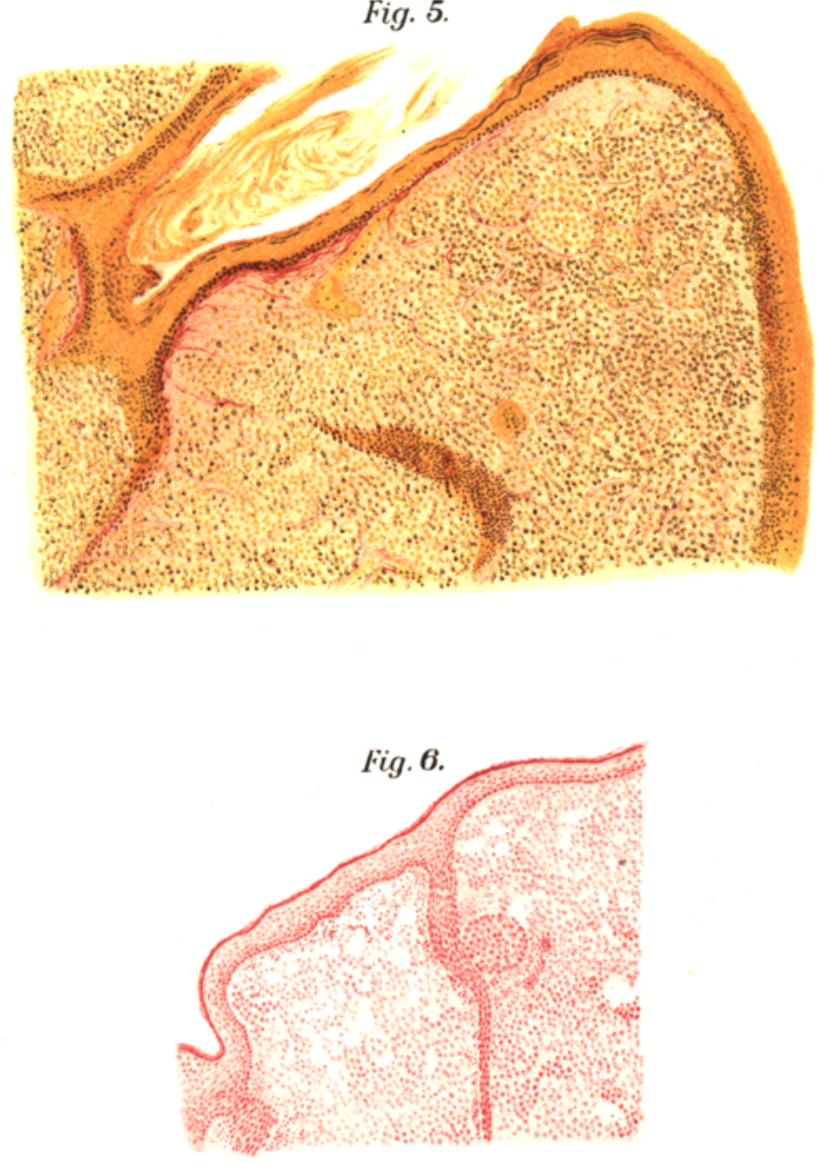

Waelsch: Cber die aus weichen Naevis entstandenen bósartigen fieschwilste. 